\title{
Node activity monitoring in heterogeneous networks using energy sensors
}

\author{
Jesus Perez, Javier Via, Luis Vielva \\ Dept. Communications Engineering \\ University of Cantabria \\ Santander, Spain \\ \{jesus.perez, javier.via, luis.vielva\}@unican.es
}

\begin{abstract}
In Heterogeneous Networks, small cells are usually deployed without operator supervision. Their proper operation highly depends on their self-adaptation capability, especially in dense HetNets where various small cells coexist in the same macrocell. This capability requires the small-cell base stations to continuously sense the radio environment, so they can dynamically adapt their operational setting (e.g. transmission power, carrier/channel selection, etc.) to the environmental conditions.

In this work we propose a new method for a small base station to monitor the activity of the rest of nodes in the macrocell. We consider a centralized sensing procedure based on the fusion of the energy levels measured by the users of the small cell at their locations. In particular, we present an efficient algorithm that enables the small base station to monitor the activity of the rest of nodes. In addition, the algorithm also provides the gain of the channels between the nodes and the users of the small cell.

Index Terms-Heterogeneous networks, cooperative sensing, energy detection, least squares.
\end{abstract}

\section{INTRODUCTION}

Heterogeneous Wireless Networks (HetNets) represent a major paradigm shift in the way cellular systems are envisioned [1]. The main idea behind HetNets is that the capacity of conventional macrocells can be significantly increased by deploying multiple low-power small base stations (SBSs) within the macrocells [2]. In HetNets the small cells coexist in the same geographical area, reusing the spectrum. So intercell interference is the biggest constraint to the potential capacity improvement of HetNets [3].

Unlike conventional base stations, SBSs are usually deployed without operator supervision, and therefore, without traditional coverage planning. A macro cell and several small cells usually coexist in the same geographical area so the traffic load in HetNets is usually random, uneven and varying in space, frequency and time. Consequently, the proper operation of a small cell highly depends on the self-optimization (SO) capability of the SBS [4].

SO requires precise knowledge of the HetNet State Information (NSI) in the SBS coverage area. NSI refers to the temporal, frequency and spatial distribution of the signals present in the cell. NSI is essentially time-varying, so it must be maintained adaptively based on dynamic spectrum sensing,

This work has been funded by the Ministerio de Economa, Industria y Competitividad (MINECO) of Spain under grant TEC2017-86921-C2-1-R (CAIMAN) and under the KERMES Network (TEC2016-81900-REDT/AEI). cooperative cognition and information exchange. In summary, SO capability requires mechanisms for continuously sensing and monitoring the radio environment around the SBS in order to improve coverage and mitigate interference from coexisting nodes in the macrocell.

\section{A. Contribution}

In this work we propose a new method for the SBSs to acquire and monitor NSI in its coverage area. A SBS usually serves a number of users within its small cell. These users can act as energy sensors by measuring the RSS (received signal strength) at their locations, and reporting it to the SBS through the uplink channels. Apart form being the fusion center, the SBS can also act as another energy sensor by measuring the RSS at its own location. The layout is depicted in figure 1 .

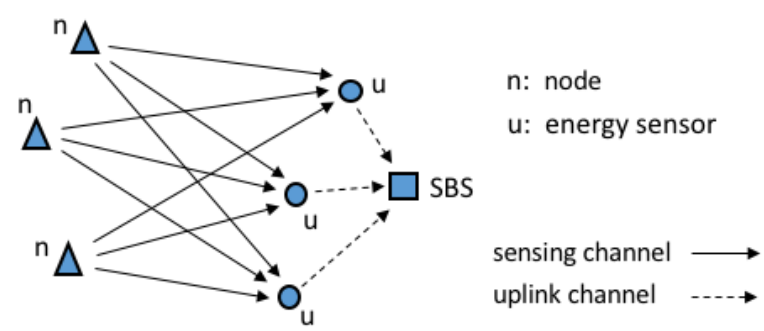

Fig. 1. NSI acquisition based on a centralized energy sensors network.

The energy levels at the sensors, in a given frequency channel, depend on which subset of nodes are active at each time. Hereafter we refer to the subset of active nodes in the channel, as channel state.

In this work we present an efficient algorithm that allows the SBS to monitor the channel state from the energy levels at the sensors. In addition the algorithm provides the gains of the sensing channels. The algorithm is able to deal with incomplete data, so it does not require the sensors to sense the channel in a synchronized way. If the sensors are moving around the cell, this information, collected over time, can be used to create space-time-frequency interference maps or dynamic network cartography of the small cell coverage area [5], [6]. 


\section{B. Related work}

This problem is related to cooperative spectrum sensing [7], [8], where the sensors are cognitive radios, equipped with energy detectors, and the fusion center is a secondary base station [9]-[12]. But the goal of spectrum sensing is to detect whether a given frequency channel is idle or occupied at a given time. Whereas in the NSI acquisition problem we aim to know what is the state of the channel, being the idle channel the particular state in which none of the nodes is transmitting. Therefore, acquiring NSI is one step further than the conventional spectrum sensing.

\section{SYSTEM MODEL}

\section{A. Channel state}

We consider a general model for the nodes activity in a given frequency channel, where more than one node can transmit simultaneously. Let $s_{k} \in\{0,1\}$ indicate the state of node $k$, with $s_{k}=1$ when it is transmitting and $s_{k}=0$ otherwise. Then, the channel state is given by the binary vector $\mathbf{s}=\left[\begin{array}{llll}s_{1} & s_{2} & \cdots & s_{K}\end{array}\right]^{T}$, where $K$ is the number of nodes operating in the frequency channel and the superscript $T$ denotes transpose. Note that $\mathbf{s}=\mathbf{0}$ is the state where none of the nodes is transmitting so the channel is idle. We denote by $\mathcal{S}$ the set of all possible channel states. Then, its cardinality $|\mathcal{S}|=2^{K}$.

\section{B. Energy detection}

Let $W$ denote the channel bandwidth. The sensors perform energy detection [13] for a time duration of $\tau$, and hence they take $M=W \tau$ signal samples during $\tau$. The normalized received energy estimate at sensor $j$ is

$$
e_{j}=\frac{2}{\eta_{j}} \sum_{m=1}^{M}\left|z_{j}[m]\right|^{2},
$$

where $z_{j}[m]$ denotes the $m$-th baseband complex signal sample and $\eta_{j}$ is the noise variance at sensor $j$. We assume that the channel state does not change during the sensing time $\tau$.

\section{Distribution of the energy estimates}

The distribution of the energy estimates depends on the channel state. Let $e_{j} \mid \mathbf{s}$ be the received energy estimate at sensor $j$ conditioned to the channel state $\mathbf{s}$. Assuming that the signals from different users are independent, $e_{j} \mid \mathbf{s}$ follows a non-central chi-square distribution with $2 M$ degrees of freedom and non-centrality parameter $\lambda_{\mathbf{s}, j}=2 M \mathbf{s}^{T} \mathbf{g}_{j}$, where $\mathbf{g}_{j}=\left[\begin{array}{llll}g_{1, j} & g_{2, j} & \cdots & g_{K, j}\end{array}\right]^{T}$, and $g_{i, j}$ is the SNR at sensor $j$ when node $i$ is the only active [11], [14].

According to the central limit theorem, if $M$ is large enough (e.g. $M \geq 20$ in practice), $e_{j} \mid \mathbf{s}$ is approximately normal distributed with mean and variance,

$$
\mathrm{E}\left[e_{j} \mid \mathbf{s}\right]=2 M+\lambda_{\mathbf{s}, j}, \quad \operatorname{Var}\left[e_{j} \mid \mathbf{s}\right]=4 M+4 \lambda_{\mathbf{s}, j} .
$$

Lets normalize the energy estimates as follows

$$
x_{j}=\frac{e_{j}-2 M}{2 M} .
$$

Therefore, under network state $\mathbf{s}$, the normalized energy $x_{j}$ will be approximately normal distributed with mean and variance given by

$$
\mathrm{E}\left[x_{j} \mid \mathbf{s}\right]=\mathbf{s}^{T} \mathbf{g}_{j}, \quad \operatorname{Var}\left[x_{j} \mid \mathbf{s}\right]=\left(1+2 \mathbf{s}^{T} \mathbf{g}_{j}\right) / M .
$$

Then, the normalized energy estimate at sensor $j$ can be written as follows,

$$
x_{j}=\mathbf{s}^{T} \mathbf{g}_{j}+r_{j},
$$

where $r_{j}$ is a zero-mean Gaussian-distributed residual with variance given in (1).

\section{Energy vectors}

Every time the sensors report their energy estimates to the SBS, it groups them in an energy vector. Let $\mathbf{x}(n)=$ $\left[x_{1}(n) x_{2}(n) \cdots x_{J}(n)\right]^{T}$ the energy vector at time $n$, where $J$ denotes the number of sensors. From (2), assuming that the network was in state $\mathbf{s}(n)$ when $\mathbf{x}(n)$ was acquired,

$$
\mathbf{x}(n)=\mathbf{G}^{T} \mathbf{s}(n)+\mathbf{r}(n),
$$

where $\mathbf{G}=\left[\mathbf{g}_{1} \mathbf{g}_{2} \cdots \mathbf{g}_{J}\right], J$ is the number of sensors and $\mathbf{r}(n)$ is the corresponding residual vector.

A complete energy vector requires all sensors to sense the channel simultaneously and to report their energy estimates to the SBS in a coordinated way. This can lead to a large overhead in the control channel. Also, if there are several channels to sense, only a subset of the sensors will be sensing a given channel at the same time. Then, we consider that the energy vectors may be incomplete because only a subset of the sensors have reported their energy estimates. The energy estimates available at the SBS can be expressed as follows,

$$
\mathbf{y}(n)=\mathbf{v}(n) \circ \mathbf{x}(n)=\mathbf{v}(n) \circ \mathbf{G}^{T} \mathbf{s}(n)+\mathbf{v}(n) \circ \mathbf{r}(n),
$$

where $\circ$ denotes Hadamart product and $\mathbf{v}(n)$ is a $J \times 1$ binary vector that indicates which sensors have computed and reported its energy level at time $n$. Therefore, $v_{j}(n)=1$ means that the energy estimate of sensor $j$ at time $n$ is available at the SBS, otherwise $v_{j}(n)=0$.

Considering $N$ consecutive time slots, the corresponding energy vectors (possibly acquired under different network states) can be grouped as follows,

$$
\mathbf{Y}=\mathbf{V} \circ \mathbf{S ~ G}+\mathbf{V} \circ \mathbf{R},
$$

where $\mathbf{Y}=[\mathbf{y}(1) \cdots \mathbf{y}(N)]^{T}, \mathbf{V}=[\mathbf{v}(1) \cdots \mathbf{v}(N)]^{T}, \mathbf{S}=$ $[\mathbf{s}(1) \cdots \mathbf{s}(N)]^{T}$ and $\mathbf{R}=[\mathbf{r}(1) \cdots \mathbf{r}(N)]^{T}$. Consequently, the sequence of energy estimates reported by sensor $j$, can be expressed as follows

$$
\mathbf{y}_{j}=\mathbf{V}_{j} \mathbf{x}_{j}=\mathbf{V}_{j} \mathbf{S} \mathbf{g}_{j}+\mathbf{V}_{j} \mathbf{r}_{j},
$$

where $\mathbf{V}_{j}=\operatorname{diag}\left(\mathbf{v}_{j}\right)$, being $\mathbf{v}_{j}$ a $N \times 1$ binary vector that indicates the time slots when sensor $j$ has reported the energy estimate to the SBS. 


\section{PROBLEM FORMULATION}

The goal is to estimate $\mathbf{S}$ and $\mathbf{G}$ from the observed energy vectors $\mathbf{Y}$. Then, we consider the following optimization problem

$$
\hat{\mathbf{S}}, \hat{\mathbf{G}}=\underset{\mathbf{S}, \mathbf{G}}{\operatorname{argmin}}\|\mathbf{Y}-\mathbf{V} \circ \mathbf{S} \mathbf{G}\|_{F}
$$

where $\|\cdot\|_{F}$ denotes the Frobenius norm. This is an optimization problem with mixed integer and continuous variables. More specifically, the rows of $\mathbf{S}$ are binary vectors from $\mathcal{S}$, whereas the entries of $\mathbf{G}$ take real positive values.

The fact that $\mathbf{S}$ is a binary matrix makes (5) different to other related problems like non-negative matrix factorization or dictionary learning.

For (5) to be well posed, $\mathbf{S}$ must be full rank. Therefore, assuming that the number of energy vectors is not less than the number or nodes $(N \geq J)$, the columns of $\mathbf{S}$ must be linear independent. A necessary condition is that all nodes have been active at least once. Otherwise, the number of effective nodes would be less than $K$.

\section{THE ALGORITHM}

We propose an alternating optimization algorithm to estimate $\mathbf{S}$ and $\mathbf{G}$ from the available energy estimates, $\mathbf{Y}$. Each iteration involves the following two successive steps:

1) First, we minimize (5) with respect to $\mathbf{S}$ keeping fixed the last estimate of the SNR matrix, $\hat{\mathbf{G}}$. The minimization can be done separately for each column of $\mathbf{S}$,

$$
\hat{\mathbf{s}}(n)=\underset{\mathbf{s} \in \mathcal{S}}{\operatorname{argmin}}\left\|\mathbf{y}(n)-\mathbf{v}(n) \circ \hat{\mathbf{G}}^{T} \mathbf{s}\right\|, \quad n=1, \ldots, N .
$$

Since $\mathbf{s}$ is a binary vector with $K$ entries, it can take $2^{K}$ different values at most. Therefore, for a moderate number of nodes, the problem can be exactly solved by means of enumeration.

2) Then, we minimize (5) with respect $\mathbf{G}$ keeping fixed the last estimate of the channel states, $\hat{\mathbf{S}}$. This minimization can be done separately for each column of G. From,(4),

$$
\hat{\mathbf{g}}_{j}=\underset{\mathbf{g} \succeq \mathbf{0}}{\operatorname{argmin}}\left\|\mathbf{y}_{j}-\mathbf{V}_{j} \hat{\mathbf{S}} \mathbf{g}\right\|, \quad j=1, \ldots, J,
$$

This is a conventional non-negative least squares problem, which is a convex optimization problem that can be solved efficiently [15].

Starting from an initial random guess for $\mathbf{G}$ (with positive entries), this two-stage optimization is repeated until there is no further change in $\hat{\mathbf{S}}$. The resulting algorithm is summarized in Algorithm 1.

Each stage reduces the value of the objective function (5), so the convergence of the algorithm is assured. However, it may converge to a local solution. Therefore, it is convenient to run the algorithm with different initial random guesses, $\hat{\mathbf{G}}^{(0)}$, and then keeping the solution with the minimum residual error

$$
\|\mathbf{R}\|_{F}^{2}=\|\mathbf{X}-\hat{\mathbf{S}} \hat{\mathbf{G}}\|_{F}^{2} .
$$

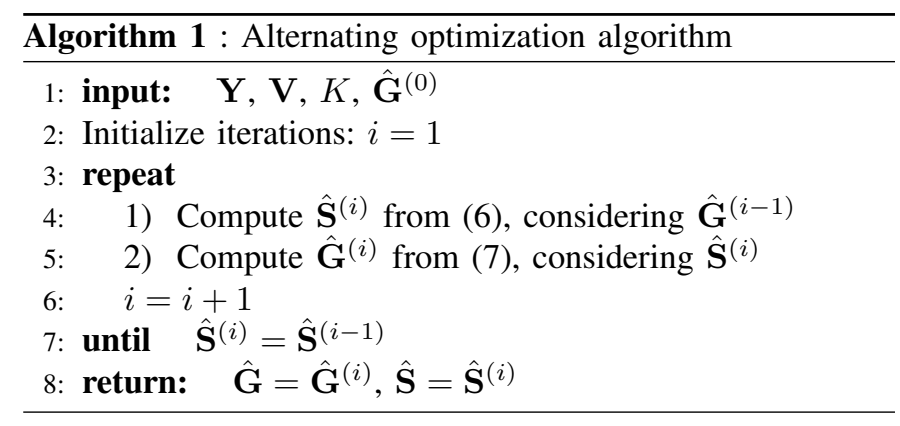

\section{Simulation Results}

\section{A. Performance metrics}

To analyze the performance of the algorithm we consider two metrics: average accuracy of the estimates $\hat{\mathbf{S}}$, and average mean squared error (MSE) of the estimates $\hat{\mathbf{G}}$.

For a given realization of $\mathbf{S}$, the accuracy of $\hat{\mathbf{S}}$ is defined as the fraction of times the nodes' state is correctly estimated,

$$
\operatorname{accuracy}(\mathbf{S}, \hat{\mathbf{S}})=\frac{1}{N K} \sum_{n=1}^{N} \sum_{k=1}^{K} \mathrm{I}\left[s_{k}(n)=\hat{s}_{k}(n)\right],
$$

where $I[\cdot]$ is the indicator function. For a given realization of $\mathbf{G}$, the MSE of $\hat{\mathbf{G}}$ is

$$
\operatorname{MSE}(\mathbf{G}, \hat{\mathbf{G}})=\frac{1}{J K}\|\mathbf{G}-\hat{\mathbf{G}}\|_{F}^{2} .
$$

In the forthcoming performance curves we average the accuracy and MSE values of 10000 independent simulations with random $\mathbf{S}$ and $\mathbf{G}$.

We compare our results, with the performance of an ideal MAP classifier with perfect knowledge of $\mathbf{G}$, so it perfectly knows the distribution of the energy estimates (1). We refer it as the clairvoyant predictor. Its performance can be considered as an upper bound for any technique solely based on RSS measures.

\section{B. Experimental setup}

Unless otherwise indicated, we have considered the following assumptions and parameter values in the simulations,

- The channel bandwidth is $W=5 \mathrm{MHz}$.

- The number of nodes is $K=3$ and the number of sensors is $J=3$.

- The channels between different pairs node-sensor are independent and identically Rayleigh distributed. In this way we average the performance over a wide variety of sensing channel conditions. We assume that the sensing channels do not change during the acquisition of the energy vectors.

- The number of signal samples for energy estimation is $M=200$, therefore, the sensing time is $\tau=\frac{M}{W}=40 \mu \mathrm{s}$.

- The number of energy vectors is $N=16$.

- The channel state can change between two consecutive energy vectors. The activity of each node is modeled as an independent and identically distributed homogeneous Markov chain with two states: inactive $\left(s_{k}=0\right)$ and 
active $\left(s_{k}=1\right)$. In the simulations we assume that the transition probability $\mathrm{P}\left(s_{k}(n)=0 \mid s_{k}(n-1)=\right.$ $0)=0.75$, whereas the transition probability $\mathrm{P}\left(s_{k}(n)=\right.$ $\left.1 \mid s_{k}(n-1)=1\right)$ is chosen so the probability that the channel is idle is $\mathrm{P}(\mathbf{s}=\mathbf{0})=0.3$, which depends on the number of nodes. Accordingly, for $K=3$ nodes, $\mathrm{P}\left(s_{k}(n)=1 \mid s_{k}(n-1)=1\right)=0.5$.

- All nodes transmit identical power and all sensors have the same noise variance. Then, the average SNR is the same for all sensors (averaging over the sensing channel realizations $\mathbf{G}$ and the nodes activity $\mathbf{S}$ ). The assumption is mainly made to facilitate the interpretability of the results. Unless otherwise indicated, we assume that $\mathrm{E}\left[g_{k, j}\right]=-3 \mathrm{~dB}, \forall k, j$.

The total number of energy estimates at the SBS is $N_{o}=\sum_{n=1}^{N} \sum_{j=1}^{J} v_{j}(n)$, which can take values in the interval $N \leq N_{o} \leq J N$. In the simulations, when $N_{o} \leq J N$, the sensors that report their energy estimates are selected randomly. Obviously, an adequate sensor selection policy would produce better results. Unless otherwise indicated, we assume that all energy estimates are available at the SBS, so $N_{o}=J N$.

\section{Results}

Figure 2 shows the average accuracy as a function of the average SNR. It can be observed that the performance loss with respect to the clairvoyant predictor is low and remains almost constant in a wide range of average SNR values. Also, the accuracy gain of increasing the average SNR is lower for higher values.

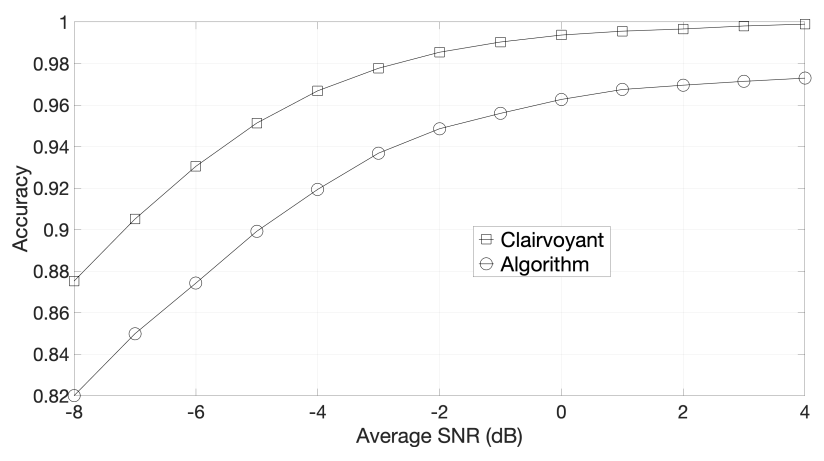

Fig. 2. Average accuracy as a function of the average SNR.

Figure 3 shows the average accuracy and average MSE curves as a function of the number of sensors $J$. As it is expected, the higher the $J$ the better the performance. The performance gap with respect to the clairvoyant predictor decreases with the number of sensors.

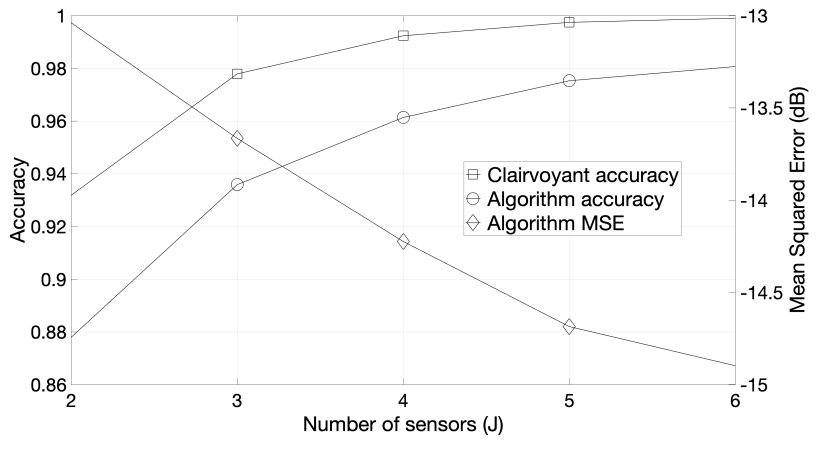

Fig. 3. Average accuracy and MSE for different number of sensors $(J)$.

Figure 4 shows the average accuracy and average MSE curves as a function of the number of energy vectors $N$. As it is expected, the higher the $N$, the lower the MSE. The average accuracy increases with $N$ but the accuracy gain of increasing $N$ is lower for higher values. Note that high values of $N$ could violate the assumption that the gains of the sensing channels $(\mathbf{G})$ remain constant during the acquisition of the energy vectors.

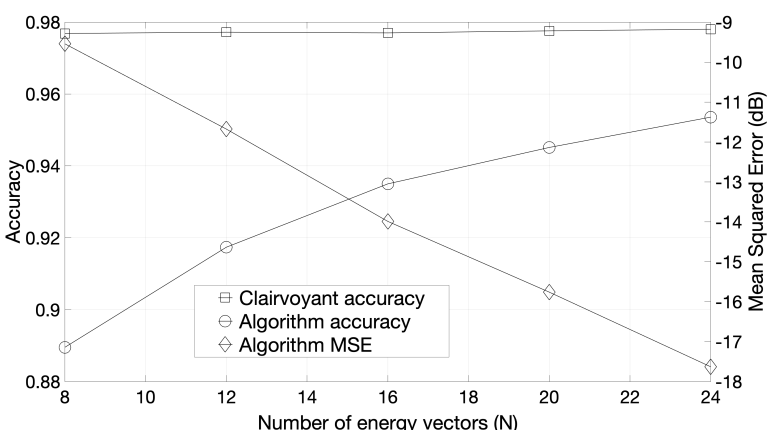

Fig. 4. Average accuracy and MSE for different number of energy vectors $(N)$.

Figure 5 shows the performance as a function of the number of sensing samples $M$. As it is expected, the performance grows with $M$. In addition, the performance gap with respect the clairvoyant predictor decreases with $M$. Note that high values of $M$ could violate the assumption that the channel state does not change during the sensing time $\tau$.

Figure 6 shows the average accuracy as a function of the number of sensors $J$ for different number of nodes $K$. In this case the average SNR is $0 \mathrm{~dB}$. Interestingly, beyond a number of sensors the average accuracy remains practically constant, so the performance increases very little with the number of sensors.

Finally, figures 7 and 8 show the accuracy as a function of the number of energy estimates $N_{o}$ for different values of average SNR and for different number of nodes $K$, respectively. In the second figure the average MSE is also shown. As it is expected, the performance grows with $N_{o}$ in all cases. In the case $N_{o}=48$ all the energy estimates are available at the SBS, whereas when $N_{o}=16$ only the energy measurement from one sensor is available at each time. 


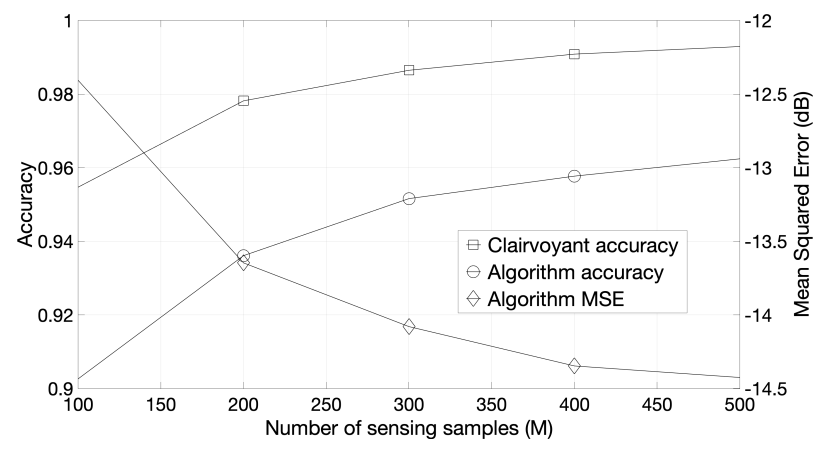

Fig. 5. Average accuracy and MSE as a function of the number of sensing samples $(M)$.

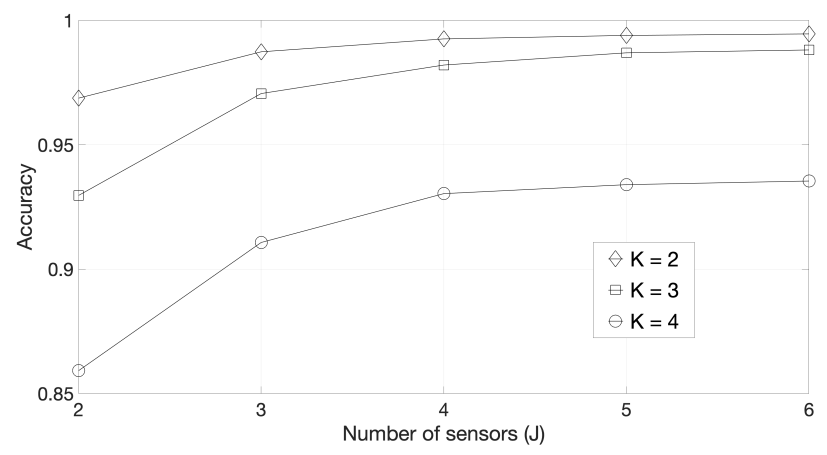

Fig. 6. Average accuracy as a function of the number of sensors for different values of nodes. Average SNR $=0 \mathrm{~dB}$.

\section{CONCLUSIONS}

In this work we have presented a novel method that allows a SBS to monitor the activity of the rest of nodes in a HetNet macrocell. It is based on a centralized network of energy sensors, where the fusion center is the SBS and the sensors are the users of the small cell. We present an algorithm that provides the subset of active nodes at each time in a given frequency channel. In addition, it estimates the channel gains from the nodes to the sensors. The algorithm is based on a simple and robust alternating optimization procedure. It is able to deal with incomplete data, so it does not require the energy sensors to be synchronized. The simulation results show that the algorithm is feasible and efficient for monitoring the activity in HetNet macrocells.

\section{REFERENCES}

[1] J. G. Andrews, "Seven ways that hetnets are a cellular paradigm shift," IEEE Communications Magazine, pp. 136-144, March 2013.

[2] X. Chu, D. Lopez-Perez, Y. Yang, and F. Gunnarsson, Eds., Heterogeneous Cellular Networks: Theory, Simulation and Deployment, Cambridge University Press, 2013.

[3] G. de la Roche M. Kountouris T. Q. S. Quek J. Zhang D. Lopez-Perez, I. Guvenc, "Enhanced intercell interference coordination challenges in heterogeneous networks," IEEE Wireless Communications, pp. 22-30, June 2011.

[4] F. Gunnarsson, "Self-organization," in Heterogeneous cellular networks. Theory, simulations and deployment, Xialy Chu, David Lopez-Perez, Yang Yang, and Fredrik Gunnarsson, Eds., chapter 6, pp. 145-178. Cambridge University Press, 2013.

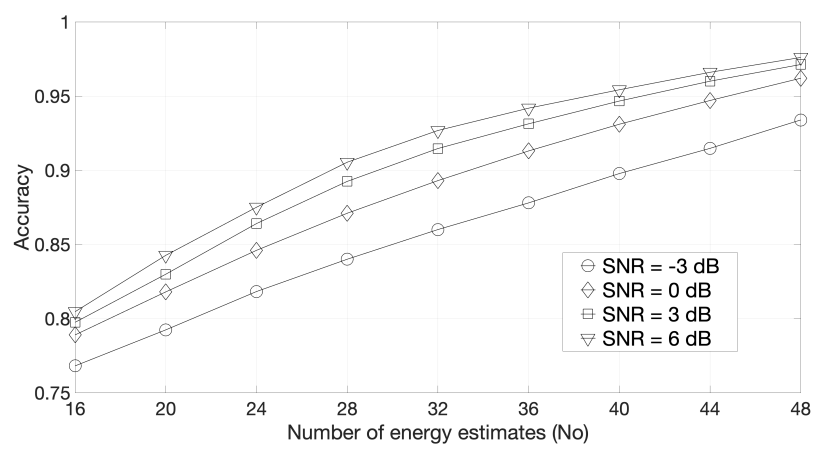

Fig. 7. Average accuracy as a function of the number of energy estimates at the SBS $\left(N_{o}\right)$ for different values of average SNR.

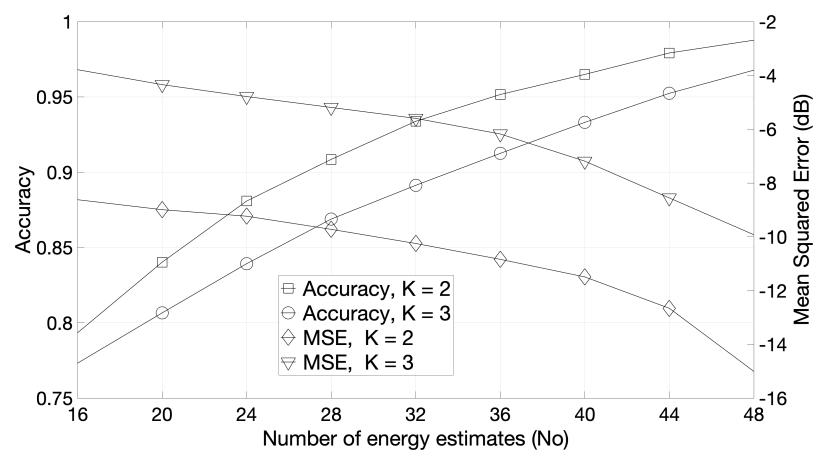

Fig. 8. Average accuracy as a function of the number of energy estimates at the SBS $\left(N_{o}\right)$ for different number of nodes $(\mathrm{K})$.

[5] G. Mateos and K. Rajawat, "Dynamic network cartography," IEEE Signal Processing Magazine, vol. 30, no. 3, pp. 129-143, May 2013.

[6] D. Romero, S. J. Kim, G. B. Giannakis, and R. Lopez-Valcarce, "Learning power spectrum maps from quantized power measurements," IEEE Transactions on Signal Processing, vol. 65, no. 10, pp. 2547-2560, May 2017.

[7] I. F. Akyildiz, B. F. Lo, and R. Balakrishnan, "Cooperative spectrum sensing in cognitive radio networks: a survey," Physical Communications, vol. 4, no. 1, pp. 40-62, March 2011.

[8] Y. Zeng, Y. C. Liang, A. T. Hoang Liu, and R. Zhang, "A review on spectrum sensing for cognitive radio: challenges and solutions," EURASIP Journal on Advances in Signal Processing, vol. 2010, no. 1, January 2010.

[9] J. Ma, G. Zhao, and Y. Li, "Soft combination and detection for cooperative spectrum sensing in cognitive radio networks," IEEE Transactions on Wireless Communications, vol. 7, no. 11, pp. 45024507, December 2008.

[10] Z. Quan, S. Cui, and A. H. Sayed, "Optimal linear cooperation for spectrum sensing in cognitive radio networks," IEEE Journal of Selected Topics in Signal Processing, vol. 2, no. 1, pp. 28-40, February 2008.

[11] K. M. Thilina, K. W. Choi, N. Saquib, and E. Hossain, "Machine learning techniques for cooperative spectrum sensing in cognitive radio networks," IEEE Journal on Selected Areas in Communications, vol. 11, no. 10, pp. 2209-2221, November 2013.

[12] J. Perez and I. Santamaria, "Adaptive clustering algorithm for cooperative spectrum sensing in mobile environments," in IEEE International Conference on Acoustics, Speech and Signal Processing (ICASSP), Calgary,Canada, April 2018, IEEE, IEEE.

[13] H. Urkowitz, "Energy detection of unknown deterministic signals," Proc. IEEE, vol. 55, pp. 523-531, April 1967.

[14] J. Ma, G. Y. Li, and B. H. Juang, "Signal processing in cognitive radio," Proceedings of the IEEE, vol. 97, no. 5, pp. 805-823, May 2009.

[15] C. L. Lawson and R. J. Hanson, Solving least squares problems, Prentice Hall, 1995. 